\title{
Adaptive OFDM for High Mobility Computer Application
}

\author{
K.V.N. Kavitha and K. Vinoth Babu
}

\begin{abstract}
OFDM based wireless systems are spectrally efficient but they are very sensitive to Doppler shift. The high mobility causes the channel to vary fast and the rapid variation of the channel can induce Inter Carrier Interference (ICI). ICI due to fast fading channel is more difficult to handle and it causes loss of orthogonality between the sub carriers. This kind of ICI can be easily reduced by increasing the sub carrier bandwidth. In this paper we proposed an Adaptive OFDM system which will use Variable Sub carrier Bandwidth (VSB) and Variable Bit Loading (VBL) based on the Doppler velocity to minimize the effect of ICI. We investigate the performance of such Adaptive OFDM system with conventional OFDM system based on throughput.
\end{abstract}

Index terms-Adaptive OFDM, ICI, VSB-VBL Technique

\section{INTRODUCTION}

OFDM has been used for wireless applications, like digital broadcasting of digital audio, digital television, and the mobile radio communication. Initially OFDM systems are targeted for fixed and low mobility applications. More recently, high mobility applications are targeted. The high mobility introduces more Doppler shift which causes loss of orthogonality between the sub carriers and introduces ICI.

In conventional OFDM systems most of the approaches to combat ICI are towards using frequency synchronization and interference cancellation. They are usually very complex and sometimes there is loss in bandwidth efficiency [1]. In the conventional OFDM system sub carrier bandwidth is kept constant for all the Doppler velocities. Authors in [1] have proposed VSB-VBL technique to select variable sub carrier bandwidth based on the Doppler velocity. The same technique is used in this paper to design Adaptive OFDM.

The organization of this article is as follows; High mobility and channel estimation error is described in section II. Section III contains the Analytical Model and section IV contains algorithm to find VSB-VBL. Results and Discussion are in section $\mathrm{V}$, which is followed by the conclusion in section VI.

Manuscript received $20^{\text {th }}$ April, 2009. This work is supported by Wireless and Networking Division of School of Electrical Sciences, VIT University, Vellore, India.

K.V.N.Kavitha, Senior Assistant professor is now with the School of Electrical Sciences, Vellore Institute of Technology, Vellore, India. (e-mail: kvnkavitha@yahoo.co.in).

K.Vinoth Babu, Assistant Professor is now with the School of Electrical Sciences, Vellore Institute of Technology, Vellore, India. (e-mail: vinothbabu.k@vit.ac.in).

\section{High Mobilty And Channel Estimation ERror}

To detect the input signal the channel needs to be known. For low mobility applications the channel is assumed to be constant within one OFDM symbol. But for high mobility applications the channel is variant within one OFDM symbol. So the channel has to be estimated from the training data or the statistics characteristics of the channel. The difference between the estimated channel and actual channel is channel estimation error. The high mobility contributes more channel estimation error which also introduces ICI.

\section{ANALYTiCAl Model}

In OFDM, IFFT is used at the transmitter side and FFT at the receiver side. The sent sequence is formed by first taking IFFT and then pre-appending CP [5]. It is given by,

$$
z_{n}=\frac{1}{N} \sum_{k=0}^{N-1} Z_{k} e^{j \frac{2 \pi k n}{N}} n=0,1, \ldots N-1
$$

Let the received sequence i.e input to FFT is denoted by $y_{0}$, $y_{1}, \ldots y_{N-1}$ Let $H_{k, n}$ denote the transfer function of channel for carrier $k$ and sample $n$ of sent signal.

Considering the Kth sub carrier at the output of FFT [5],

$$
\begin{aligned}
Y_{K} & =\sum_{n=0}^{N-1} y_{n} e^{-j \frac{2 \pi}{N} K n} \\
Y_{K} & =\sum_{n=0}^{N-1} \frac{1}{N} \sum_{k=0}^{N-1} Z_{K} H_{k, n} e^{\frac{j 2 \pi\left(k-K^{\prime}\right) n}{N}} \\
Y_{K} & =\sum_{k=0}^{N-1} Z_{K} \sum_{n=0}^{N-1} \frac{1}{N} H_{k, n} e^{\frac{j 2 \pi\left(k-K^{\prime}\right) n}{N}}
\end{aligned}
$$

Here the channel for the $K$ th sub carrier during the reception of one OFDM symbol is assumed to vary in a linear fashion [5].

$$
H_{K, n}=\overline{H_{K}}+\left(n-\frac{N-1}{2}\right) \frac{H_{K}^{\prime}}{N}
$$

Where $H_{K}^{\prime}$ is the channel change during the symbol and $H_{K}$ is the average channel experienced during the OFDM symbol.Consequently, the ICI on sub carrier $K$ caused by the symbol sent on sub carrier $K+L$ is given by [5] ,

$$
Y_{K, K+L} \approx Z_{K+L} H_{K+L}^{\prime} \frac{1}{j 2 \pi L}
$$

In case Jake's model is assumed for the Doppler spectrum the well known formula for the ICI power caused by Doppler is obtained, 
$P_{I C I}=\frac{\pi^{2}}{6} f_{d}^{2}$

Where $f_{d}=\frac{f_{D}}{\Delta f}$ is normalized Doppler i.e actual

Doppler divided by sub carrier bandwidth.

When there is only one path the received signal is $y_{i}=h_{i} * z_{i}+w_{i} \quad 0 \leq i \leq N$

Where $w_{i}$ is the white Gaussian noise.

The estimate of the input is

$$
z_{i}=\frac{y_{i}}{\hat{h}_{i}}=z_{i}+\frac{z_{i} \cdot e_{i}}{\hat{h_{i}}}+\frac{w_{i}}{\hat{h_{i}}} \quad 0 \leq i \leq N
$$

Where $\hat{h}_{i}$ is the estimate $h_{i}$ and $e_{i}$ is the channel estimation error.

The signal to interference plus noise ratio is

$$
\operatorname{SINR}=v_{r x}\left[K, \Delta f_{m}\right]=\frac{z_{i}^{2}}{\left|\frac{z_{i} \cdot e_{i}}{\hat{h}_{i}}+\frac{w_{i}}{\hat{h}_{i}}\right|^{2}}
$$

\section{AlgORITHM To FInd VSB-VBL}

Here we propose an algorithm to select sub carrier bandwidth and bit load per sub carrier based on Doppler velocity to achieve target BER.

The following steps are executed in sequence.

1) Select one sub carrier bandwidth from the available options.

2) Evaluate (10), i.e. SINR at each sub carrier for the selected sub carrier spacing.

3) Bit load per estimate [1], [3] is calculated using (11).

$$
b_{L}\left(K, \Delta f_{m}\right)=2\left\lfloor\frac{1}{2} \log _{2}\left(1-\frac{1.6}{\ln \left(\frac{b 0 r e q}{0.2}\right)}\right) v_{r x}\left[K, \Delta f_{m}\right]\right\rfloor
$$

where $L$.$\rfloor operator is the floor operation. In the above$ expression $b 0$ req is the target BER which is to be satisfied.

4) Calculate $B E R[1],[4]$ by using the following expression

$$
b_{0}\left(K, \Delta f_{m}\right)=0.2 e^{\frac{-1.6 v_{r x}\left[K, \Delta f_{m}\right]}{2^{b L\left(K, \Delta f_{m}\right)-1}}}
$$

5) Store the value of BER along with the value of sub carrier band width and associated bit loads per sub carrier.

6) Repeat all the above steps for all possible values of sub carrier bandwidth.

7) Execute the following to find best sub carrier bandwidth[1],[2]

$$
\Delta f_{\text {chosen }}=\min \left[\operatorname{BER}\left(\Delta f_{m}\right)\right]
$$

\section{RESUlts AND Discussions}

In this paper we use Jake's model for each path of time varying Rayleigh Fading channel. The channel is modelled as three tap delay line. The carrier frequency is $5 \mathrm{GHz}$ and bandwidth of one channel is $20 \mathrm{MHz}$. The target BER is kept at $10^{-3}$. The number of bits that can be loaded on a sub carrier are $0,2,4,6,8,10$, where ' 0 ' means no transmission. Four different conventional Fixed Sub carrier Bandwidth systems are taken for the comparison purpose. The system with 2048 sub carrier has $\Delta f_{m}=2.4 \mathrm{kHz}(\mathrm{C}-2048)$. The system with 1024 sub carrier has $\Delta f_{m}=4.88 \mathrm{kHz}(\mathrm{C}-1024)$. The system with 512 sub carrier has $\Delta f_{m}=9.77 \mathrm{kHz}(\mathrm{C}-512)$. The system with 256 sub carrier has $\Delta f_{m}=19.531 \mathrm{kHz}(\mathrm{C}-256)$. The Adaptive OFDM system is named as A-OFDM in the figures.

Fig.1. is the plot between SINR and sub carrier bandwidth at SNR of $60 \mathrm{~dB}$. Here four different velocities $(50,100,150,200 \mathrm{kmph})$ are taken for the analysis. It can be seen from the figure that SINR improves with increasing sub carrier bandwidth for a given Doppler velocity.

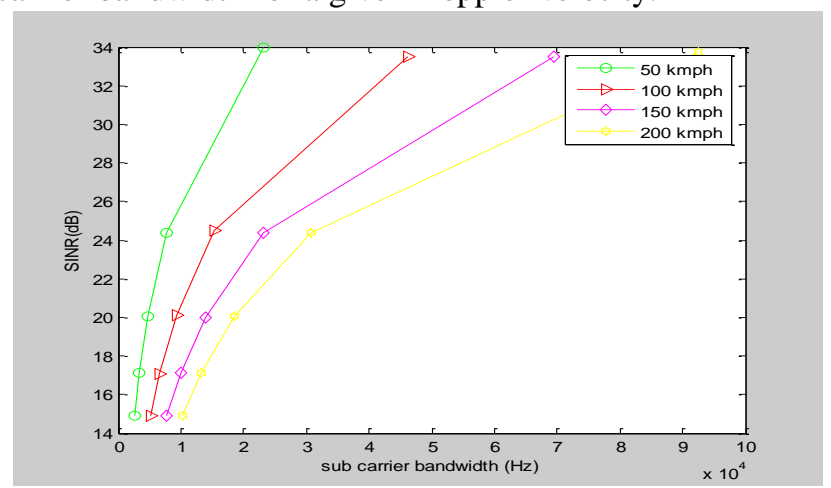

Fig.1 SINR vs. Sub carrier bandwidth at SNR of $60 \mathrm{~dB}$

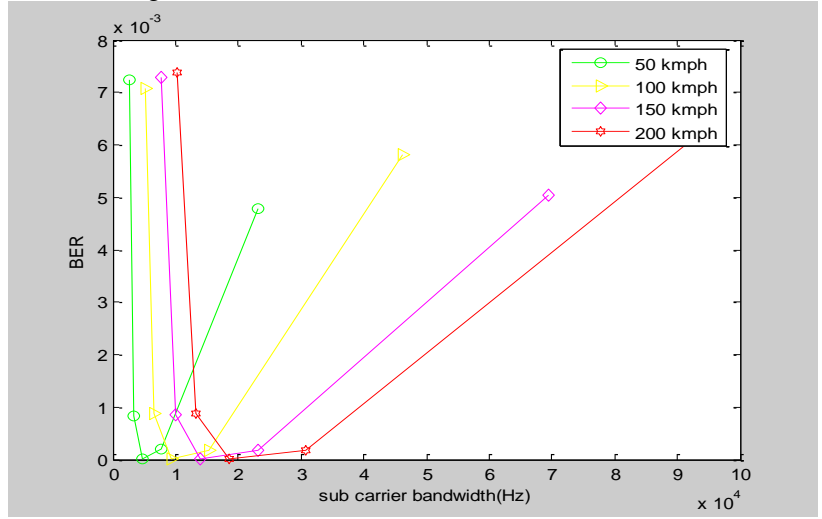

Fig.2 BER vs. Sub carrier bandwidth at SNR of $60 \mathrm{~dB}$

Fig.2. is plot between BER and sub carrier bandwidth at SNR of $60 \mathrm{~dB}$. Each curve is for a particular velocity. The BER value decreases upto certain bandwidth then keeps on increasing. The sub carrier bandwidth at which BER value is minimum that bandwidth is taken as variable bandwidth for that particular doppler velocity.

Fig.3. shows the average sub carrier bandwidth selected by Adaptive OFDM system based on VSB-VBL technique for different values of velocity at a received SNR of $60 \mathrm{~dB}$. 


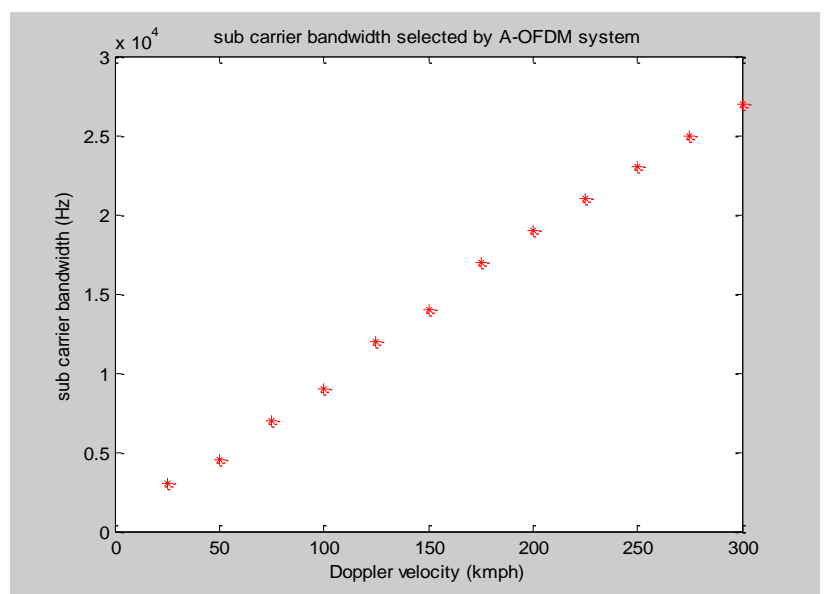

Fig.3 Sub carrier bandwidth selected by VSB system

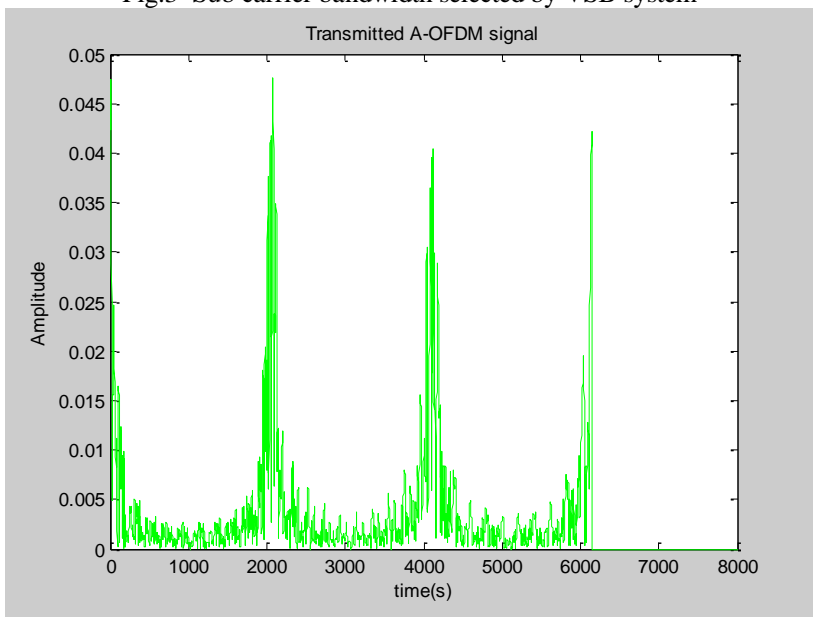

Fig.4 Transmitted A-OFDM signal for $50 \mathrm{kmph}$ Doppler velocity

Fig.4. shows the transmitted A-OFDM signal for the Doppler velocity of $50 \mathrm{kmph}$. A-OFDM has longer symbol duration for low Doppler velocities. The sub carrier bandwidth selected by A-OFDM system for the Doppler velocity of $50 \mathrm{kmph}$ is $5 \mathrm{kHz}$. Fig.5. shows the spectrum generated for A-OFDM at $50 \mathrm{kmph}$ Doppler velocity.

Fig.6. shows the transmitted A-OFDM signal for the Doppler velocity of $200 \mathrm{kmph}$. A-OFDM has shorter symbol duration for high Doppler velocities. The sub carrier bandwidth selected by A-OFDM system for the Doppler velocity of $200 \mathrm{kmph}$ is $19 \mathrm{kHz}$. Fig.7. shows the spectrum generated for A-OFDM at $200 \mathrm{kmph}$ Doppler velocity.

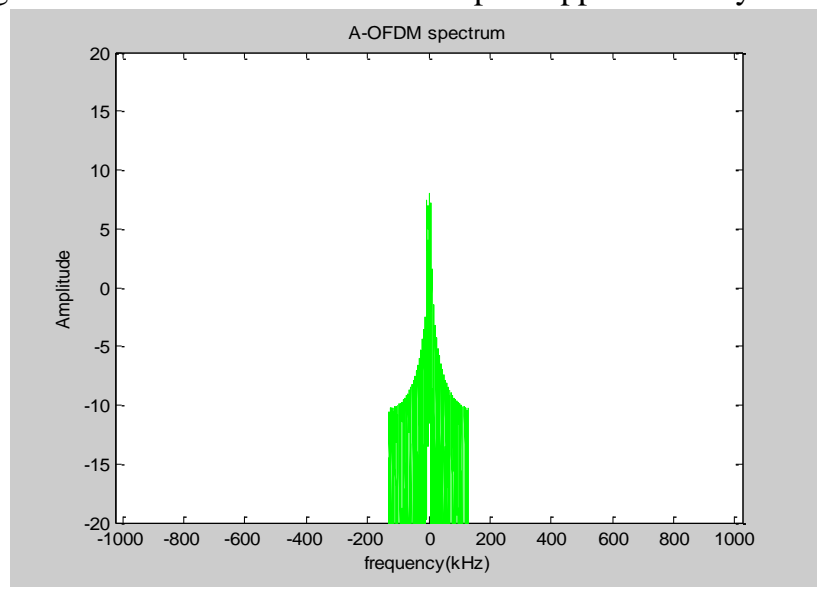

Fig.5 A-OFDM Spectrum for $50 \mathrm{kmph}$ Doppler velocity

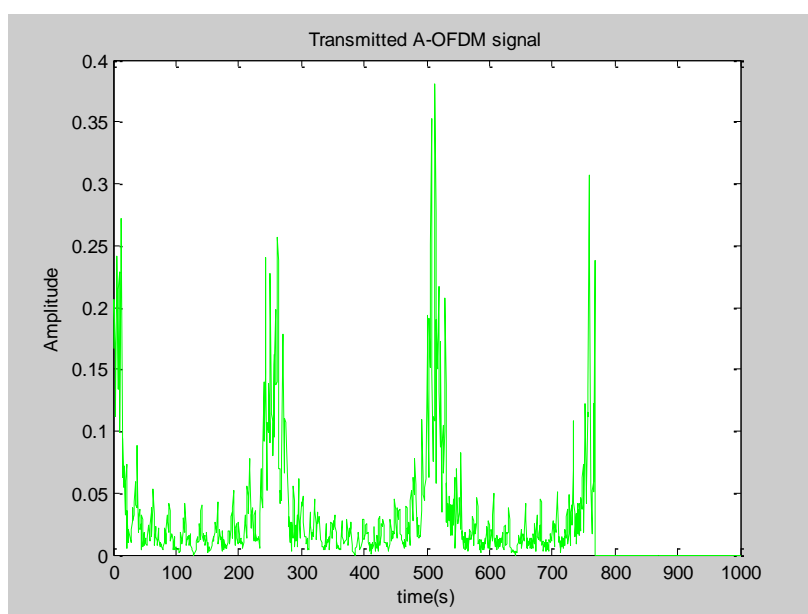

Fig.6 Transmitted A-OFDM signal for 200 kmph Doppler velocity

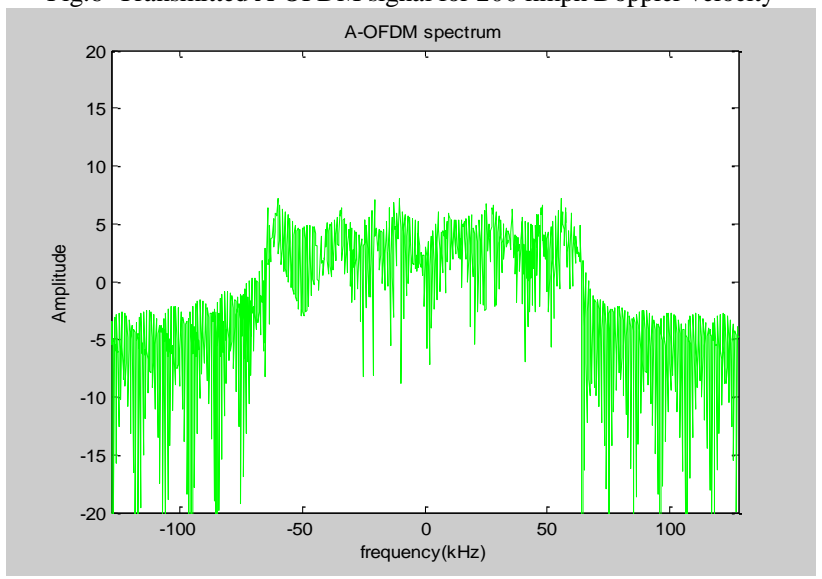

Fig.7 A-OFDM Spectrum for $200 \mathrm{kmph}$ Doppler velocity

Fig.8. is the Throughput comparision of A-OFDM and various C-OFDM systems at SNR of $60 \mathrm{~dB}$. Upto the doppler velocity $200 \mathrm{kmph}$ the C-256 OFDM system has higher throughput when compared to all other systems. Then upto $100 \mathrm{kmph}$ C-512 OFDM system has high throughput when compared to A-OFDM,C-1024,C-2048 systems. Then upto $50 \mathrm{kmph}$ the C-1024 OFDM system has high throughput when compared to A-OFDM and C-2048 systems. When the doppler velocity reaches $200 \mathrm{kmph}$ throughput value of all C-OFDM systems (including C-256) becomes lower when compared to A-OFDM system. So A-OFDM system has optimal throughput performance .

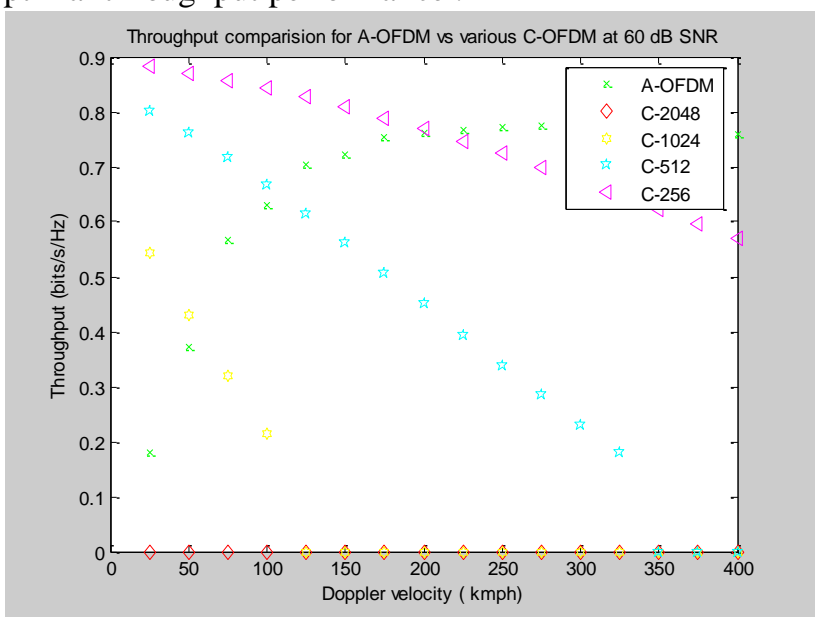

Fig.8 Throughput comparison of A-OFDM and various C- OFDM systems at SNR of $60 \mathrm{~dB}$. 


\section{CONCLUSIONS}

It has been found that the Conventional OFDM system is optimum over a small range of Doppler velocity and it is not well suited for high mobility applications. Adaptive OFDM system has optimum throughput performance for low and high mobility conditions. It improves throughput [2.95\% to $23.41 \%$ ] when compared to conventional OFDM systems for high mobility applications.

\section{REFERENCES}

[1] S. S. Das , Elisabeth De Carvalho and Ramjee Prasad., "Performance Analysis of OFDM with Adaptive Sub Carrier Bandwidth "in Proc. IEEE ICC 2008, vol. 7,NO.4, APRIL2008, pp. 1117-1122.

[2] S. S. Das ,E.De Carvalho, Ramjee Prasad., "Variable Sub-Carrier Bandwidths in OFDM Systems "in Proc. IEEE ICC 2007, pp. 1866-1870.

[3] S. T. Chung and A. J. Goldsmith, "Degrees of freedom in adaptive modulation: a unified view," IEEE Trans. Commun., vol. 49, no. 1, pp.1561-1571, Sept. 2001.

[4] S. S. Das, Muhammad Imadur Rahman1, Frank H.P. Fitzek., "Multi rate orthogonal frequency division multiplexing,"in Proc. IEEE ICC 2005, vol. 4, May 2005, pp. 2588-2592.

[5] Mike Faulkner., "Low complex ICI cancellation for improving Doppler performance in OFDM systems." in Proc. IEEE 2006

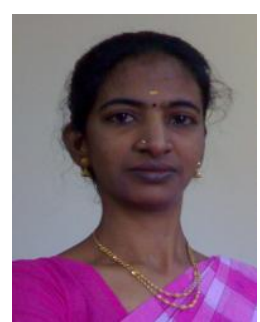

K.V.N.Kavitha received B.E Degree from Madras University, India in 1998 and M.E Degree in Applied Electronics from Anna University, India.

She is now working as Senior Assistant Professor in School of Electrical Sciences in VIT University, Vellore, India. She has published several papers in various International Conferences like ICINT, ICMLC in the areas of OFDM .She has also published papers in the International Journals like IIUM, IARIA,ICFAI.

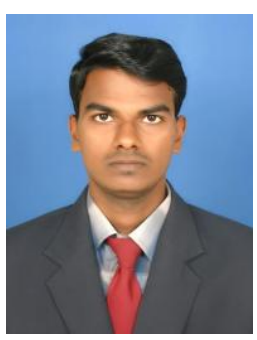

K. Vinoth Babu received B.E Degree in Electronics and Communication Engineering from Anna University, Chennai, India in 2005 and M.Tech Degree in Communication Engineering from VIT University, Vellore, India.

He is now working as Assistant Professor in School of Electrical Sciences in VIT University, Vellore ,India. His major areas of interest include CDMA and OFDM. He has published several papers in various International Conferences like ICINT2009, ICSTE 2009, ICMLC 2009 in the areas of OFDM and APH. 